\title{
Clinical Usefulness of Electronic Drug-Drug Interaction Checking in the Care of Cardiovascular Surgery Inpatients
}

\author{
A.B. Taegtmeyer ${ }^{a, b} \quad$ G.A. Kullak-Ublick ${ }^{a} \quad$ N. Widmer ${ }^{c} \quad$ V. Falk W. Jetter $^{\mathrm{a}}$ \\ ${ }^{a}$ Department of Clinical Pharmacology and Toxicology, University Hospital Zurich, Zurich, and ${ }^{b}$ Department of \\ Clinical Pharmacology and Toxicology, University Hospital Basel, Basel, and ' Division of Cardiac and Vascular \\ Surgery, University Hospital Zurich, Zurich, Switzerland
}

\section{Key Words}

Medication safety $\cdot$ Interaction checking $\cdot$ Clinical decision support system $\cdot$ Cardiovascular surgery

\begin{abstract}
Objectives: Drug-related problems (DRPs) are events or circumstances involving drug therapy that actually or potentially interfere with desired health outcomes. This study tested the applicability of clinical decision support software in identifying and managing DRPs among cardiovascular surgery inpatients. Methods: Two clinical pharmacologists attended ward rounds on a low-dependency cardiovascular surgery ward every 2 weeks over a 7-month period. Three hundred and three patients were assessed. On average, patients received 17 scheduled and 'as required' medicines. DRPs were identified 'manually' via assessment of electronic prescription charts and patient records and 'electronically' using clinical decision support software (Pharmavista ${ }^{\circledR}$ ). The numbers of alerts for optimizing medication safety generated by the two methods were compared. Results: Manual checking identified 346 DRPs leading to 346 alerts in 201 patients (overall 1.1 alerts/patient). Relevant interactions accounted for $44 \%$ of DRPs detected by clinical pharmacologists. Clinical decision support software, which could only
\end{abstract}

report interactions, however, generated 1,370 alerts (average 4.5 alerts/patient). Only 147 (11\%) drug-drug interaction alerts were identical to those identified by manual checking; the remaining $89 \%$ were considered not clinically relevant. Conclusions: Compared to identification of DRPs by clinical pharmacologists, the clinical decision support software performed poorly due to over-alerting and inability to assess for problems not caused by drug-drug interactions.

Copyright ๑ 2012 S. Karger AG, Basel

\section{Introduction}

Computerized physician order entry (CPOE) is becoming more widespread in the care of cardiovascular surgery inpatients. While CPOE is associated with a reduction in drug-prescribing errors [1] - primarily through prevention of misinterpretation of handwritten prescriptions - new problems related to the electronic nature of drug prescribing itself have emerged with time [2]. Furthermore, CPOE cannot prevent drug-related problems (DRPs) which arise, for example, due to concurrent morbidities, renal or hepatic dysfunction, or drug-drug interactions. Clinical pharmacology and/or clinical pharmacy services can provide valuable input in

\section{KARGER}

Fax +41613061234 E-Mail karger@karger.ch www.karger.com
(C) 2012 S. Karger AG, Basel

0008-6312/12/1234-0219\$38.00/0

Accessible online at:

www.karger.com/crd
Alexander Jetter, $\mathrm{MD}$

Department of Clinical Pharmacology and Toxicology, University Hospital Zurich

Rämistrasse 100

CH-8091 Zurich (Switzerland)

E-Mail alexander.jetter@usz.ch 
identifying DRPs and providing suggestions as to how they might be avoided. Such services, however, are laborintensive and costly. An alternative is the use of clinical decision support software (CDSS) which can be integrated into the electronic prescribing process. However, the applicability of such a system in the care of cardiovascular surgery inpatients is not known. The purpose of this study was to determine the applicability of a simple computerized decision support system which assessed for drug-drug interactions (the commonest type of CDSS in Europe) in a group of such patients by comparing its performance with that of clinical pharmacologists in identifying DRPs.

\section{Methods and Materials}

Ward rounds of pre- and postoperative cardiovascular surgery patients were attended every second week by two clinical pharmacologists over a 7-month period from November 2010 to June 2011. This study period was chosen because the treating surgeons and physicians did not change during this time. The setting was a ward without intensive care or high-dependency beds. All patients were cared for using integrated electronic medical records (with electronic prescribing). An additional feature of the electronic prescription chart was the electronic drug interaction check CDSS supplied by Pharmavista ${ }^{\circledR}$ (e-mediat AG, Bern, Switzerland) [3]. When requested to do so by the prescriber, this program assessed potential drug-drug interactions and graded these according to the required intervention (based on the operational classification of drug interactions by Hansten et al. [4]). The CDSS did not flag up potential problems automatically. Such alerts are known as 'noninterruptive' alerts and differ from 'interruptive' alerts which require acknowledgement from the prescriber of the awareness of the DRP usually in the form of a mouse click. It was not known how often the interaction check software was used by the prescribing surgeons. Other than the voluntary drug interaction check, no electronic clinical decision support (regarding dosing, for example) was embodied in the electronic prescription chart.

Clinical pharmacologists assessed the electronic prescription charts for the presence of drug-related issues. Internet-based databases including Swiss, German, and US product information, PubMed, the Pharmavista ${ }^{\circledR}$ tool [3], and the Micromedex ${ }^{\circledR}$ Healthcare Series (Thomson Reuters, Greenwood Village, Col., USA) [5] were used.

DRPs were defined according to the Pharmaceutical Care Network Europe (PCNE) classification for drug-related problems, version 6.2 (revised on January 14, 2010), which defines a DRP as 'an event or circumstance involving drug therapy that actually or potentially interferes with desired health outcomes' [6]. Drug-related issues included all DRPs and instances where specific information regarding drug therapy (such as the pharmacokinetics of intravenously or orally administered antibiotics) was requested by the treating surgeons. Proposals for optimizing drug safety were then given to the treating surgeons face-to-face on the ward round.
For the purposes of analysis, DRPs were classified according to a simplified PCNE system which defined the drug-related issues as a lack of treatment effect (P1 of PCNE version 6.2), an adverse drug event (P2), and provision of information. Underlying causes of a lack of treatment effect or an adverse drug event were classified as resulting from an interaction (C1.3), inappropriate dosing (C3), contraindication (C1.1), inappropriate timing of administration (C5.1), inappropriate duplication of therapeutic group or active ingredient (C1.4), unclear prescription (including problems arising from electronic prescribing) (C6.2), need for therapeutic drug monitoring (C3.5), known side effect of the drug, and failure to correctly document drug allergies and suboptimal choice of drug form (C2.1) or drug (C1.1).

Data are presented as numbers and percentages. The average number of drugs prescribed per patient was determined from a randomly selected subgroup of 30 patients. Similarly, data on the number of drug prescriptions which CDSS was unable to recognize was collected for 7 ward rounds and used to estimate the overall percentage of drug prescriptions which could not be included in the automated analysis for drug-drug interactions. A positive predictive value calculation was performed using clinical pharmacologists' judgments as the gold standard.

\section{Results}

The electronic prescription charts of 303 patients were assessed. On average, each patient had 17 different medications prescribed on a regular and 'as required' basis. Of these prescriptions, approximately $4.5 \%$ could not be recognized by CDSS as they had been entered into the electronic drug chart as 'free text' fields.

The types and frequencies of drug-related issues are shown in table 1. Potential adverse drug events were the most common $(72 \%)$, followed by potential lack of treatment effect (15\%), actual adverse drug events (6\%) and provision of information pertaining to drug prescriptions as requested by the treating surgeons (4\%). Drugdrug interactions were the underlying cause of 153 DRPs (44\% of all DRPs). The 5 most common drug-drug interactions were paracetamol in combination with an enzyme inducer (such as rifampicin or pheny toin, $n=9$ ), the combination of rifampicin and an opiate analgesic $(\mathrm{n}=$ 7), the combination of polyvalent cations with fluoroquinolones $(n=6)$ or levothyroxine $(n=6)$, and the combination of amiodarone with statins $(\mathrm{n}=5)$. Formally contraindicated drug-drug combinations were the combination of amiodarone with domperidone, an antiemetic associated with QTc prolongation $(\mathrm{n}=4)$. These latter cases, however, did not cause any manifest adverse drug events.

CDSS identified 147 of these 153 interactions, in addition to reporting on an additional 1,223 interactions 


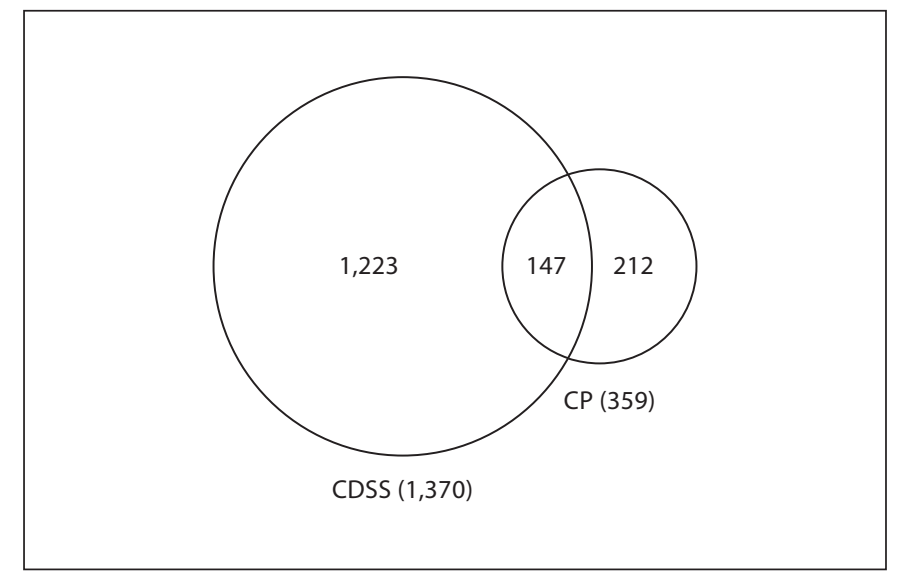

Fig. 1. Area-proportional Venn diagram of alerts generated by the CDSS and by clinical pharmacologists (CP). Figures are absolute numbers.

which were not judged clinically relevant by the clinical pharmacologists (fig. 1). In addition to this 'over-alerting', whereby only $11 \%$ of computer-generated alerts were clinically applicable, $61 \%$ of all drug-related issues could not be identified by this form of CDSS, therefore representing a simultaneous gross 'under-alerting. The positive predictive value of CDSS for interactions was 0.107 when considering clinical pharmacologists' judgments to be the 'gold standard'.

A small but relevant number of DRPs were related to the CPOE system itself $(n=9)$, as shown in the footnote to table 1 .

\section{Discussion}

While the incidence of DRPs in this group of cardiovascular surgery inpatients was less than that found in other studies of hospitalized patients [7], drug-drug interactions were the underlying cause for the majority of the detected DRPs. This was higher than found in a previous study of medical inpatients at the same hospital (33\%) [8] and likely reflects the more frequent use of drugs such as amiodarone and rifampicin in our patient population. While CDSS was able to detect nearly all of the drug-drug interactions seen by clinical pharmacologists, it also generated a large number of over-alerts $(89 \%$ of CDSS-generated alerts were not judged to be clinically relevant) in addition to being unable to alert on dosing adaptation, drug allergies, and other contraindications.

Clinical Decision Support in

Cardiovascular Surgery
Table 1. Drug-related issues ( $\mathrm{n}=359$, of which 346 were DRPs) and, where applicable, their underlying causes in 303 cardiovascular surgery inpatients

\begin{tabular}{|c|c|c|}
\hline $\begin{array}{l}\text { Drug-related issue/ } \\
\text { underlying cause }\end{array}$ & $\begin{array}{l}\text { Number } \\
\text { (\% of total) }\end{array}$ & $\begin{array}{l}\text { Number } \\
\text { identified } \\
\text { by CDSS }\end{array}$ \\
\hline Lack of treatment effect & 0 & 0 \\
\hline \multicolumn{3}{|l|}{ Adverse drug event } \\
\hline Known side effect of drug & $21(5.8)^{\mathrm{b}}$ & 0 \\
\hline Interaction & $1(0.3)$ & $1(0.3 / 0.1)$ \\
\hline \multicolumn{3}{|l|}{ Potential lack of treatment effect } \\
\hline Interaction & $44(12.2)$ & $40(11.1 / 2.9)$ \\
\hline Inappropriate dose & $9(2.5)^{c}$ & 0 \\
\hline Time of administration & $1(0.3)$ & 0 \\
\hline \multicolumn{3}{|l|}{ Potential adverse drug event } \\
\hline Known side effect of drug & $33(9.1)$ & 0 \\
\hline Interaction & $104(29.0)$ & $102(28.4 / 7.4)$ \\
\hline Time of administration & $5(1.4)$ & 0 \\
\hline Drug choice ${ }^{\mathrm{d}}$ & $17(4.7)$ & 0 \\
\hline Inappropriate dose & $45(12.5)^{\mathrm{e}}$ & 0 \\
\hline Contraindication & $28(7.8)$ & $4(1.1 / 0.3)$ \\
\hline Need for therapeutic & & \\
\hline drug monitoring & $3(0.8)$ & 0 \\
\hline Prescription unclear & $11(3.0)$ & 0 \\
\hline Duplication of therapeutic class & $15(4.2)$ & 0 \\
\hline Documentation of allergies & $7(1.9)^{\mathrm{f}}$ & 0 \\
\hline Information given & $15(4.2)$ & 0 \\
\hline
\end{tabular}

a $\%$ of all drug-related issues/\% of total interactions found by CDSS $(\mathrm{n}=1,370)$.

b Two adverse drug events reported to the authorities (one case of paradoxical reaction to lorazepam and one case of levodopa withdrawal symptoms).

${ }^{c}$ Seven due to incorrect prescription of low-molecular-weight heparin as 1 unit instead of 1 prefilled syringe - a problem facilitated by the electronic prescribing process.

$\mathrm{d}$ Two due to incorrect prescription of apomorphine instead of morphine for analgesia - a problem facilitated by the electronic prescribing process per se.

${ }^{\mathrm{e}}$ Fifteen instances where dosing exceeded the maximum licensed dose and 5 instances where dose adjustment for intermittent hemodialysis was indicated; the remainder was due to impaired renal or hepatic function and in response to therapeutic drug monitoring results.

${ }^{f}$ One instance where a drug was administered despite the patient having a documented allergy; however, no adverse drug event occurred.

The implications of these findings are threefold. First, in order to be clinically applicable, CDSS should be optimized to detect all relevant DRPs. Such systems are, however, expensive and still under development. Secondly, we found a failure to detect all clinically relevant drug-drug

Cardiology 2012;123:219-222 
interactions despite the large number of alerts generated by CDSS. In this study, the reasons why CDSS was unable to identify 6 relevant drug-drug interactions were an inability to recognize drug prescriptions entered as 'free text' (and not chosen from drop-down menus) and the exceeding of the CDSS's computational capacity in one case. These limitations of CDSS should be addressed and eliminated in future software development. Thirdly, the large number of over-alerts seen in this and other studies $[9,10]$ is cause for concern as clinicians are more likely to ignore all alerts when only 1 out of 10 alerts is relevant, thereby deriving no benefit for their patients from the CDSS. In the recent study of CDSS-generated drug-drug interaction alerts by Seidling et al. [9], only $1.4 \%$ of noninterruptive alerts were accepted. For 'interruptive' alerts the authors identified the frequency of the alert, the quality of the message displayed, the alert level, and the contextual setting (e.g. inpatients and prescriptions involving drugs with dose-dependent toxicity) as being associated with increased acceptance [9].

The present study has limitations. The judgments of the two clinical pharmacologists were taken as the 'gold standard' for the purposes of determining the performance of CDSS in this setting but they may have been incomplete or biased. However, there are no established 'gold standards' in this field. For a simple 10-category system for classifying DRPs, the inter-rater agreement was found to be 0.68 [11]. The CDSS system used here is one which is more widespread in Europe than in the USA, where systems capable of alerting on DRPs not solely arising from drug-drug interactions are commonly in place. Whether the findings of our study are more widely applicable to other patient settings, other clinical pharmacology and clinical pharmacist services, and other prescribers merits further study.

\section{Conclusions}

Taken together, our findings show that drug-drug interaction CDSS in its current form is unlikely to be of assistance to surgeons treating cardiovascular surgery inpatients due to a combination of over-alerting and the high number and complexity of DRPs which go beyond drug-drug interactions. Monitoring of the prescribed drug regimen by a clinical pharmacologist remains an important measure towards optimizing medication safety in cardiovascular surgery patients until both $\mathrm{CPOE}$ and CDSS are optimized.

\section{Conflict of Interest}

All authors have no conflicts of interest to disclose relevant to the contents of this paper.

\section{References}

1 van Doormaal JE, van den Bemt PM, Zaal RJ, Egberts AC, Lenderink BW, Kosterink JG, Haaijer-Ruskamp FM, Mol PG: The influence that electronic prescribing has on medication errors and preventable adverse drug events: an interrupted time-series study. J Am Med Inform Assoc 2009;16:816-825.

$\checkmark 2$ Redwood S, Rajakumar A, Hodson J, Coleman JJ: Does the implementation of an electronic prescribing system create unintended medication errors? A study of the sociotechnical context through the analysis of reported medication incidents. BMC Med Inform Decis Mak 2011;11:29.

3 Pharmavista: http://www.pharmavista.ch/ content/default.aspx (accessed January 15, 2012).

$\checkmark 4$ Hansten PD, Horn JR, Hazlet TK: ORCA: OpeRational ClassificAtion of drug interactions. J Am Pharm Assoc (Wash) 2001;41: 161-165.
5 Micromedex $^{\circledR}$ Healthcare Series. Greenwood Village, Thomson Reuters, ed expired 2011.

6 Pharmaceutical Care Network Europe Foundation classification for drug-related problems (revised January 14, 2010) V6.2. 2010. http://www.pcne.org/sig/drp/documents/ PCNE classification V6-2.pdf.

7 Blix HS, Viktil KK, Reikvam A, Moger TA, Hjemaas BJ, Pretsch P, Vraalsen TF, Walseth EK: The majority of hospitalised patients have drug-related problems: results from a prospective study in general hospitals. Eur J Clin Pharmacol 2004;60:651-658.

-8 Taegtmeyer AB, Curkovic I, Rufibach K, Corti N, Battegay E, Kullak-Ublick GA: Electronic prescribing increases uptake of clinical pharmacologists' recommendations in the hospital setting. Br J Clin Pharmacol 2011;72:958-964.
-9 Seidling HM, Phansalkar S, Seger DL, Shaykevich S, Haefeli WE, Bates DW: Factors influencing alert acceptance: a novel approach for predicting the success of clinical decision support. J Am Med Inform Assoc 2011;18:479-484

10 Frölich T, Zorina O, Fontana AO, KullakUblick GA, Vollenweider A, Russmann S: Evaluation of medication safety in the discharge medication of 509 surgical inpatients using electronic prescription support software and an extended operational interaction classification. Eur J Clin Pharmacol 2011;67:1273-1282.

11 Hohmann C, Eickhoff C, Klotz JM, Schulz M, Radziwill R: Development of a classification system for drug-related problems in the hospital setting (APS-Doc) and assessment of the inter-rater reliability. J Clin Pharm Ther 2012;37:276-281. 